\title{
Representation of Numerical Data on a Pair of Variables by a Polynomial Curve Expressed in the Simplest Form
}

\author{
Dhritikesh Chakrabarty \\ Department of Statistics, Handique Girls' College, Guwahati - 781001, Assam, India. \\ e-mail: dhritikesh.c@ rediffmail.com,dhritikeshchakrabarty@gmail.com
}

\begin{abstract}
A formula has been derived for representing a set of numerical data on a pair of variables by a polynomial expressed in the simplest form due to its necessity in the approach of interpolation namely the approach which consists of the representation of numerical data by a polynomial in the simplest form and then to compute the value of the dependent variable corresponding to any given value of the independent variable from the polynomial leads to the necessity of a method/formula for representing a given set of numerical data on a pair of variables by the polynomial as mentioned. This paper describes the derivation of this formula with one numerical example of its application.
\end{abstract}

Key Words: 1;Pair of variables 2;polynomial in simplest form 3; representation of numerical data 4; interpolation.

\section{Introduction}

Interpolation is a technique of estimating approximately the value of the dependent variable corresponding to a value of the independent variable lying between its two extreme values on the basis of the given values of the independent and the dependent variables [Hummel, 1947; Erdos \& Turan, 1938; Neale \& Sommerville, 1924; Traub, 1964; et al]. A number of interpolation formulae namely Newton's forward interpolation formula, Newton's backward interpolation formula, Lagrange's interpolation formula, Newton's divided difference interpolation formula, Newton's central difference interpolation formula, Stirlings formula, Bessel's formula and some others are available in the literature of numerical analysis [Bathe \& Wilson, 1976; Jan, 1930; Hummel, 1947; Quadling, 1966; Whittaker \&, 1967; Mills, 1977; Revers \& Michael Bull, 2000; et al.]. The existing formulae for interpolation are nothing but expressions of the polynomial represented by the observed values expressed in different forms. However, each of these forms is complicated. Moreover, for 
obtaining the value of the polynomial at some value of the independent variable by the existing formulae all the observed numerical values, in addition to the value of the independent variable, are to be used in the formulae. As a result, if it is wanted to evaluate the values of the polynomial (i.e. the dependent variable) corresponding to a number of values of the independent variable by any existing formulae, it is required to use all the observed numerical values in the formulae in the computation of every evaluation. One can get rid of this repeated computation if a polynomial in the (simplest) form

$$
y=a_{0}+a_{1} x+a_{2} x^{2}+a_{3} x^{3} \ldots \ldots \ldots \ldots \ldots+a_{n} x^{n}
$$

where $x \& y$ are variables and

$$
a_{0}, a_{1}, a_{2}, a_{3}, \ldots \ldots \ldots \ldots, a_{n}
$$

can be determined and may represent the given numerical data. In this case, the value of the dependent variable corresponding to any value of the independent variable can be directly calculated from the polynomial. This method of interpolation namely the approach which consists of the representation of numerical data by a polynomial in the simplest form and then to compute the value of the dependent variable corresponding to any given value of the independent variable from the polynomial leads to the necessity of a method/formula for representing a given set of numerical data on a pair of variables by the polynomial as mentioned. Attempt has here been made on searching for a formula for obtaining the simplest form of the polynomial, as mentioned above, that can represent a set of numerical data on a pair of variables. This paper describes the derivation of this formula with one numerical example of its application.

\section{Representation of Numerical Data by Polynomial Curve}

Let the dependent variable $Y$ assumes the values

$$
y_{0}, y_{1}, y_{2}, \ldots \ldots \ldots \ldots \ldots, y_{n}
$$

corresponding to the respective values

$$
x_{0}, x_{1}, x_{2}, \ldots \ldots, x_{n}
$$

of the independent variable $X$.

Then

$$
\left(x_{0}, y_{0}\right),\left(x_{1}, y_{1}\right),\left(x_{2}, y_{2}\right),\left(x_{3}, y_{3}\right), \ldots \ldots \ldots \ldots \ldots,\left(x_{n}, y_{n}\right)
$$

will be $(n+1)$ points in $X-Y$ plane.

The objective is to represent these $(n+1)$ points by a polynomial of the form 


$$
y=f(x)=a_{0}+a_{1} x+a_{2} x^{2}+a_{3} x^{3} \ldots \ldots \ldots \ldots \ldots+a_{n} x^{n}, a_{n} \neq 0
$$

where $a_{0}, a_{1}, a_{2}, \ldots \ldots \ldots \ldots \ldots, a_{n}$ are constants (i.e. independent of $x$ ).

\section{Case-1 (Linear Curve):}

First, let us represent the two points

$$
\left(x_{0}, y_{0}\right),\left(x_{1}, y_{1}\right)
$$

by a linear curve defined by

$$
y=a_{0}+a_{1} x
$$

where $a_{0} \& a_{1}$ are the parameters to be determined from the two points.

The linear curve described by equation (2.2) can be expressed as

$$
\begin{aligned}
y & =a_{0}+a_{1}\left(x-x_{0}\right)+\left\{a_{1} x-a_{1}\left(x-x_{0}\right)\right\} \\
& =\left(a_{0}+a_{1} x_{0}\right)+a_{1}\left(x-x_{0}\right)
\end{aligned}
$$

This means, the linear curve defined by equation (2.2) assumes the form

$$
y=A_{0}+A_{1}\left(x-x_{0}\right)
$$

where $A_{0}=a_{0}+a_{1} x_{0} \& A_{0}=a_{1}$

are some constants (i.e. free from $\mathrm{x}$ ), to be determined from the two points

$$
\left(x_{0}, y_{0}\right),\left(x_{1}, y_{1}\right)
$$

\section{Case-2 (Quadratic Curve):}

Next, let us represent the three points

$$
\left(x_{0}, y_{0}\right),\left(x_{1}, y_{1}\right),\left(x_{2}, y_{2}\right)
$$

by a quadratic curve defined by

$$
y=a_{0}+a_{1} x+a_{2} x^{2}
$$

where $a_{0}, a_{1} \& a_{2}$ are the parameters to be determined from the three points.

The quadratic curve described by equation (2.4) can be expressed as

$$
\begin{aligned}
y=a_{0}+ & a_{1} x+a_{2}\left(x-x_{0}\right)\left(x-x_{1}\right)+\left\{a_{2} x^{2}-a_{2}\left(x-x_{0}\right)\left(x-x_{1}\right)\right\} \\
& =\left(a_{0}-a_{2} x_{0} x_{1}\right)+\left\{a_{1}+a_{2}\left(x_{0}+x_{1}\right)\right\} x+a_{2}\left(x-x_{0}\right)\left(x-x_{1}\right)
\end{aligned}
$$

This is of the form

$$
y=a_{0}{ }^{\prime}+a_{1}{ }^{\prime} x+a_{2}{ }^{\prime}\left(x-x_{0}\right)\left(x-x_{1}\right)
$$


where $a_{0}{ }^{\prime}=a_{0}$ 焵 $a_{2} x_{0} x_{1}$,

$$
\begin{aligned}
a_{1}{ }^{\prime} & =a_{1}+a_{2}\left(x_{0}+x_{1}\right) \\
\& a_{2}{ }^{\prime} & =a_{2}
\end{aligned}
$$

are some constants (i.e. free from $x$ ).

Now, the term

$$
a_{0}^{\prime}+a_{1}{ }^{\prime} x
$$

is a linear function of $x$.

Thus as shown in the Case-1, it assumes the form described by equation (2.3).

Hence, the quadratic curve defined by equation (2.4) assumes the form

$$
y=A_{0}+A_{1}\left(x-x_{0}\right)+A_{2}\left(x-x_{0}\right)\left(x-x_{1}\right)
$$

for some other constants $A_{0}, A_{1} \& A_{2}$ to be determined from the three points

$$
\left(x_{0}, y_{0}\right),\left(x_{1}, y_{1}\right),\left(x_{2}, y_{2}\right)
$$

\section{Case-3 (Cubic Curve):}

In a similar manner, let us represent the four points

$$
\left(x_{0}, y_{0}\right),\left(x_{1}, y_{1}\right),\left(x_{2}, y_{2}\right),\left(x_{3}, y_{3}\right)
$$

by a cubic curve defined by

$$
y=a_{0}+a_{1} x+a_{2} x^{2}+a_{3} x^{3}
$$

where $a_{0}, a_{1}, a_{2} \& a_{3}$ are the parameters.

The cubic curve defined by equation (2.6) can be expressed as

$$
\begin{aligned}
y= & a_{0}+a_{1} x+a_{2} x^{2}+a_{3}\left(x-x_{0}\right)\left(x-x_{1}\right)\left(x-x_{2}\right)+\left\{a_{3} x^{3}-a_{3}\left(x-x_{0}\right)\left(x-x_{1}\right)\left(x-x_{2}\right)\right\} \\
= & \left(a_{0}+a_{3} x_{0} x_{1} x_{2}\right)+\left\{a_{1}-a_{3}\left(x_{0} x_{1}+x_{0} x_{2}+x_{1} x_{2}\right)\right\} x+\left\{a_{2}+a_{3}\left(x_{0}+x_{1}+x_{2}\right)\right\} x^{2} \\
& +a_{3}\left(x-x_{0}\right)\left(x-x_{1}\right)\left(x-x_{2}\right)
\end{aligned}
$$

This is of the form

$$
y=a_{0}{ }^{\prime}+a_{1}{ }^{\prime} x+a_{2}{ }^{\prime} x^{2}+a_{3}{ }^{\prime}\left(x-x_{0}\right)\left(x-x_{1}\right)\left(x-x_{2}\right)
$$

where $\quad a_{0}{ }^{\prime}=a_{0}+a_{3} x_{0} x_{1} x_{2}$,

$$
\begin{aligned}
a_{1}{ }^{\prime} & =a_{1} \text { 焵 } a_{3}\left(x_{0} x_{1}+x_{0} x_{2}+x_{1} x_{2}\right), \\
a_{2}^{\prime} & =a_{2}+a_{3}\left(x_{0}+x_{1}+x_{2}\right. \\
\& a_{3}{ }^{\prime} & =a_{3}
\end{aligned}
$$


are some constants (i.e. free from $x$ ).

Now, the term

$$
a_{0}^{\prime}+a_{1}^{\prime} x+a_{2}^{\prime} x^{2}
$$

is a quadratic function of $x$.

Thus as shown in the Case-2, it assumes the form described by equation (2.5).

Hence, the cubic curve defined by equation (2.6) assumes the form

$$
y=A_{0}+A_{1}\left(x-x_{0}\right)+A_{2}\left(x-x_{0}\right)\left(x-x_{1}\right)+A_{3}\left(x-x_{0}\right)\left(x-x_{1}\right)\left(x-x_{2}\right)
$$

for some other constants $A_{0}, A_{1}, A_{2} \& A_{3}$ to be determined from the four points

$$
\left(x_{0}, y_{0}\right),\left(x_{1}, y_{1}\right),\left(x_{2}, y_{2}\right),\left(x_{3}, y_{3}\right)
$$

\section{Case-4 (General Polynomial Curve):}

Continuing the similar process, as applied in the earlier cases, one can obtain that the $n^{\text {th }}$ degree polynomial curve defined by equation $(2.1)$ that represents the $(n+1)$ pairs

$$
\left(x_{0}, y_{0}\right),\left(x_{1}, y_{1}\right),\left(x_{2}, y_{2}\right),\left(x_{3}, y_{3}\right), \ldots \ldots \ldots \ldots \ldots,\left(x_{n}, y_{n}\right)
$$

of values assumes the form

$$
\begin{aligned}
y= & A_{0}+A_{1}\left(x-x_{0}\right)+A_{2}\left(x-x_{0}\right)\left(x-x_{1}\right)+A_{3}\left(x-x_{0}\right)\left(x-x_{1}\right)\left(x-x_{2}\right) \\
& +\ldots \ldots \ldots \ldots \ldots \ldots+A_{n}\left(x-x_{0}\right)\left(x-x_{1}\right)\left(x-x_{2}\right) \ldots \ldots \ldots \ldots \ldots\left(x-x_{n-1}\right)
\end{aligned}
$$

for some constants $A_{0}, A_{1}, A_{2}, A_{3}, \ldots \ldots \ldots \ldots \ldots \ldots, A_{n}$ to be determined from the given $(n$ +1 ) pairs of numerical data.

Now, the task is to express these $(n+1)$ constants of the equation given by $(2.8)$ in terms of

$$
\left(x_{0}, y_{0}\right),\left(x_{1}, y_{1}\right),\left(x_{2}, y_{2}\right),\left(x_{3}, y_{3}\right), \ldots \ldots \ldots \ldots \ldots,\left(x_{n}, y_{n}\right) \text {. }
$$

For this one can think of applying the following conditions:

$$
\begin{aligned}
& \text { when } x=x_{0} \text { then } y=y_{0}, \\
& \text { when } x=x_{1} \text { then } y=y_{1}, \\
& \text { when } x=x_{2} \text { then } y=y_{2}, \\
& \text { when } x=x_{3} \text { then } y=y_{3},
\end{aligned}
$$

when $x=x_{n}$ then $y=y_{n}$. 
Applying the condition

$$
\text { when } \boldsymbol{x}=x_{0} \text { then } y=y_{0}
$$

in (2.8), one can obtain that $A_{0}=y_{0}$

Hence (2.8) becomes,

$$
\begin{aligned}
y= & y_{0}+A_{1}\left(x-x_{0}\right)+A_{2}\left(x-x_{0}\right)\left(x-x_{1}\right)+A_{3}\left(x-x_{0}\right)\left(x-x_{1}\right)\left(x-x_{2}\right) \\
& +\ldots \ldots \ldots \ldots \ldots \ldots+A_{n}\left(x-x_{0}\right)\left(x-x_{1}\right)\left(x-x_{2}\right) \ldots \ldots \ldots \ldots \ldots\left(x-x_{n-1}\right)
\end{aligned}
$$

Again applying the condition

$$
\text { when } x=x_{1} \text { then } y=y_{1}
$$

in (2.9), one can obtain that

$$
A_{1}=\frac{\left(Y_{1}-y_{0}\right)}{\left(x_{1}-x_{0}\right)}
$$

Hence (2.8) becomes,

$$
\begin{aligned}
y= & y_{0}+\frac{\left(y_{1}-y_{0}\right)}{\left(x_{1}-x_{0}\right)}\left(x-x_{0}\right)+A_{2}\left(x-x_{0}\right)\left(x-x_{1}\right)+A_{3}\left(x-x_{0}\right)\left(x-x_{1}\right)\left(x-x_{2}\right) \\
& +\ldots \ldots \ldots \ldots \ldots \ldots+A_{n}\left(x-x_{0}\right)\left(x-x_{1}\right)\left(x-x_{2}\right) \ldots \ldots \ldots \ldots \ldots\left(x-x_{n-1}\right)
\end{aligned}
$$

i.e. $y=\frac{\left(x-x_{1}\right)}{\left(x_{\sigma}-x_{1}\right)} y_{0}+\frac{\left(x-x_{0}\right)}{\left(x_{1}-x_{0}\right)} y_{1}+A_{2}\left(x-x_{0}\right)\left(x-x_{1}\right)+A_{3}\left(x-x_{0}\right)\left(x-x_{1}\right)\left(x-x_{2}\right)$

$$
+\ldots \ldots \ldots \ldots+A_{n}\left(x-x_{0}\right)\left(x-x_{1}\right)\left(x-x_{2}\right) \ldots \ldots \ldots\left(x-x_{n-1}\right)
$$

Similarly, applying the condition

$$
\text { when } x=x_{2} \text { then } y=y_{2}
$$

in (2.10), one can obtain that

$$
A_{2}=\frac{\left(y_{2}-y_{0}\right)}{\left(x_{2} x_{0}\right)\left(x_{2}-x_{1}\right)}+\frac{\left(y_{1}-y_{0}\right)}{\left(x_{1}-x_{0}\right)\left(x_{1}-x_{2}\right)}
$$

Hence (2.10) becomes,

$$
\begin{aligned}
y= & \frac{\left(x-x_{1}\right)}{\left(x_{\sigma} x_{1}\right)} y_{0}+\frac{\left(x-x_{0}\right)}{\left(x_{1}-x_{0}\right)} y_{1}+\left\{\frac{\left(y_{2}-y_{0}\right)}{\left(x_{2}-x_{0}\right)\left(x_{2}-x_{1}\right)}+\frac{\left(y_{1}-y_{0}\right)}{\left(x_{1}-x_{0}\right)\left(x_{1}-x_{2}\right)}\right\}\left(x-x_{0}\right)\left(x-x_{1}\right) \\
& +A_{3}\left(x-x_{0}\right)\left(x-x_{1}\right)\left(x-x_{2}\right)+\ldots \ldots \ldots \ldots \ldots \ldots \ldots \ldots \ldots \ldots \ldots \ldots \ldots \ldots \ldots \ldots \ldots \ldots \ldots \ldots \ldots \ldots \\
& +A_{n}\left(x-x_{0}\right)\left(x-x_{1}\right)\left(x-x_{2}\right) \ldots \ldots \ldots \ldots \ldots
\end{aligned}
$$

i.e. $y=\frac{\left(x-x_{1}\right)\left(x-x_{2}\right)}{\left(x_{0}-x_{1}\right)\left(x_{0}-x_{2}\right)} y_{0}+\frac{\left(x-x_{0}\right)\left(x-x_{2}\right)}{\left(x_{1}-x_{0}\right)\left(x_{1}-x_{2}\right)} y_{1}+\frac{\left(x-x_{0}\right)\left(x-x_{1}\right)}{\left(x_{2}-x_{0}\right)\left(x_{2}-x_{1}\right)} y_{2}$

$+A_{3}\left(x-x_{0}\right)\left(x-x_{1}\right)\left(x-x_{2}\right)+$

$+A_{n}\left(x-x_{0}\right)\left(x-x_{1}\right)\left(x-x_{2}\right) \ldots \ldots \ldots \ldots\left(x-x_{n-1}\right)$ 
On completion of the applications of the other conditions successively, finally one obtains that

$$
\begin{aligned}
& y=\frac{\left(x-x_{1}\right)\left(x-x_{2}\right)\left(x-x_{1}\right) \operatorname{mam}\left(x-x_{\mathbb{N}-1}\right)\left(x-x_{\mathbb{N}}\right)}{\left.\left(x_{0}-x_{1}\right)\left(x_{0}-x_{2}\right)\left(x_{0}-x_{1}\right) \operatorname{mom}\left(x_{0}-x_{\mathbb{N}-1}\right)\left(x_{0}-x_{\mathbb{N}}\right)\right)} y_{0} \\
& +\frac{\left(x-x_{0}\right)\left(x-x_{2}\right)\left(x-x_{2}\right) \operatorname{mom}\left(x-x_{w-1}\right)\left(x-x_{w}\right)}{\left(x_{1}-x_{0}\right)\left(x_{1}-x_{2}\right)\left(x_{1}-x_{1}\right)_{\text {mamo }}\left(x_{1}-x_{\mathbb{W}-1}\right)\left(x_{1}-x_{w}\right)} y_{1} \\
& +\frac{\left(x-x_{0}\right)\left(x-x_{1}\right)\left(x-x_{4}\right) \operatorname{mom}\left(x-x_{w-1}\right)\left(x-x_{w}\right)}{\left(x_{2}-x_{0}\right)\left(x_{2}-x_{1}\right)\left(x_{2}-x_{1}\right) \operatorname{moma}\left(x_{2}-x_{\mathbb{N}-1}\right)\left(x_{2}-x_{m}\right)} y_{2} \\
& +\frac{\left(x-x_{0}\right)\left(x-x_{1}\right)\left(x-x_{2}\right) \text { ormo }\left(x-x_{i}-1\right)\left(x-x_{i}+1\right) \operatorname{mom}\left(x-x_{m}\right)}{\left(x_{i}-x_{0}\right)\left(x_{i}-x_{1}\right)\left(x_{i}-x_{2}\right)-\operatorname{mom}\left(x_{i}-x_{i}-1\right)\left(x_{i}-x_{i}+1\right) \operatorname{mom}\left(x_{i}-x_{i}\right)} y_{i}
\end{aligned}
$$

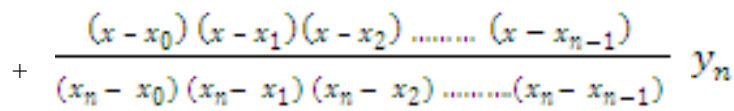

This is one mathematical form of the polynomial curve to represent the $(n+1)$ points

$$
\left(x_{0}, y_{0}\right),\left(x_{1}, y_{1}\right),\left(x_{2}, y_{2}\right),\left(x_{3}, y_{3}\right),
$$
,$\left(x_{n,}, y_{n}\right)$.

Now the task is to express it in the form defined by equation (2.1).

Equation (2.11) can be written as

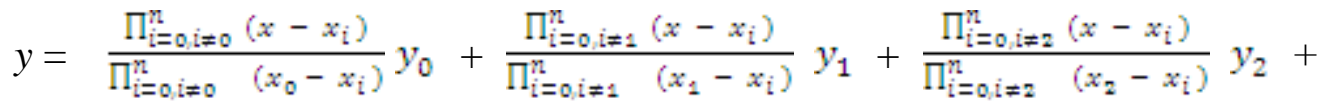

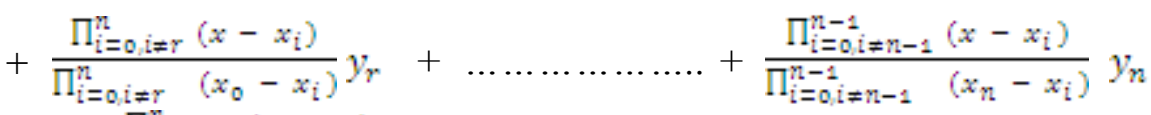

$$
\begin{aligned}
& =\sum_{r=0}^{n}\left\{\frac{\prod_{i=0, i \neq Y}^{\mathrm{n}}\left(x-x_{i}\right)}{\prod_{i=0, i \neq Y}^{\mathrm{n}}\left(x_{Y}-x_{i}\right)}\right\} y_{r} \\
& =\sum_{r=0}^{n}\left\{\frac{y_{T}}{\prod_{i=0 i \neq Y}^{P}\left(x_{Y}-x_{i}\right)}\right\} \prod_{i=0, i \neq r}^{r}\left(x-x_{i}\right)
\end{aligned}
$$

Writing

$$
\begin{aligned}
& C_{0}=\frac{y_{0}}{\mathbb{M}_{i=0}^{\mathrm{n}}\left(x_{0}-x_{i}\right)}, \\
& C_{1}=\frac{y_{1}}{\prod_{i=0}^{\mathrm{n}}\left(x_{1}-x_{i}\right)}, \\
& i \neq 0, i \neq 1 \\
& C_{2}=\frac{y_{2}}{\prod_{i=0}^{\mathrm{n}}\left(x_{2}-x_{i}\right)}, \\
& i \neq 0, i \neq 2
\end{aligned}
$$




$$
\begin{aligned}
& \left.C_{n}=\frac{y_{m}}{\prod_{i=0}^{M}\left(x_{n}-x_{i}\right)}\right) \\
& i \neq n
\end{aligned}
$$

i.e.

$$
C_{r}=\frac{y_{Y}}{\prod_{i=0}^{\mathrm{M}}\left(x_{Y}-x_{i}\right)},(i=0,1,2,3, \ldots \ldots ., n),
$$

the expression (2.12) can be written as

$$
\begin{aligned}
& \begin{array}{ccc}
y=C_{0} & \left\{\prod_{i=0}^{n}\left(x-x_{i}\right)\right\}+C_{1}\left\{\prod_{i=0}^{n n}\left(x-x_{i}\right)\right\} & +C_{2}\left\{\prod_{i=0}^{n}\left(x-x_{i}\right)\right\}+ \\
i \neq 0 & i \neq 1
\end{array} \\
& +C_{r}\left\{\prod _ { i = 0 } ^ { n } \left(\begin{array}{c}
\left(x-x_{i}\right) \\
i \neq r
\end{array}+\ldots \ldots \ldots++C_{n}\left\{\prod _ { i = 0 } ^ { n } \left(\begin{array}{c}
\left(x-x_{i}\right) \\
i \neq n
\end{array}\right.\right.\right.\right.
\end{aligned}
$$

Now,

$$
\begin{aligned}
& \prod_{i=0}^{n}\left(x-x_{i}\right)=\left(x-x_{1}\right)\left(x-x_{2}\right)\left(x-x_{3}\right) \ldots \ldots \ldots \ldots\left(x-x_{n}\right) \\
& i \neq 0 \\
& =x^{n}+\left(\sum_{i=0}^{n} x_{i}\right) x^{n-1}+\left(\sum_{i=0}^{n} \quad \sum_{j=0}^{n} x_{i} x_{j}\right) x^{n-2}+ \\
& i \neq 0 \quad i \neq 0 \quad j \neq 0 \\
& \left(\sum_{i=0}^{n} \sum_{j=0}^{n} \sum_{k=0}^{n} x_{i} x_{j} x_{k}\right) x^{n-3}+ \\
& i \neq 0 \quad j \neq 0 \quad k \neq 0 \\
& +\left(\sum_{i_{1}=0}^{n} \quad \sum_{i_{2}=0}^{n} \ldots \ldots \ldots \sum_{i_{r-1}=0}^{n} x_{i_{1}} x_{i_{2}} \ldots \ldots x_{i_{r-1}}\right) x^{n-r} \\
& i_{1} \neq 0, i_{2} \neq 0, \ldots \ldots, i_{r-1} \neq 0 \\
& +\left(x_{1} x_{2} x_{3} \ldots \ldots \ldots \ldots x_{n}\right)
\end{aligned}
$$

Similarly,

$$
\begin{aligned}
& \prod_{i=0, i \neq 1}^{n}\left(x-x_{i}\right)=\left(x-x_{0}\right)\left(x-x_{2}\right)\left(x-x_{3}\right) \ldots \ldots \ldots\left(x-x_{n}\right) \\
& =x^{n}+\left(\sum_{i=0}^{n} x_{i}\right) x^{n-1}+\left(\sum_{i=0}^{n} \sum_{j=0}^{n} x_{i} x_{j}\right) x^{n-2}+ \\
& i \neq 1 \quad i \neq 1 \quad j \neq 1 \\
& \left(\sum_{i=0}^{n} \quad \sum_{j=0}^{n} \quad \sum_{k=0}^{n} x_{i} x_{j} x_{k}\right) x^{n-3}+
\end{aligned}
$$

$i \neq 1 \quad j \neq 1 \quad k \neq 1$

$$
\begin{aligned}
+\left(\sum_{i_{1}=0}^{n} \sum_{i_{2}=0}^{n} \ldots \ldots \ldots \sum_{i_{r-1}=0}^{n} x_{i_{1}} x_{i_{2}} \ldots \ldots . x_{i_{r-1}}\right) x^{n-r} \\
i_{1} \neq 0, i_{2} \neq 0, \ldots \ldots, i_{r-1} \neq 0 \\
+\ldots \ldots \ldots \ldots \ldots \ldots+\left(x_{1} x_{2} x_{3} \ldots \ldots \ldots \ldots x_{n}\right)
\end{aligned}
$$

$$
\prod_{i=0, i \neq 2}^{n}\left(x-x_{i}\right)=\left(x-x_{0}\right)\left(x-x_{1}\right) \ldots \ldots \ldots\left(x-x_{n}\right)
$$




$$
\begin{aligned}
& =x^{n}+\left(\sum_{i=0}^{n} x_{i}\right) x^{n-1}+\left(\sum_{i=0}^{n} \sum_{j=0}^{n} x_{i} x_{j}\right) x^{n-2}+ \\
& i \neq 2 \quad i \neq 2 \quad j \neq 2 \\
& \left(\sum_{i=0}^{n} \sum_{j=0}^{n} \sum_{k=0}^{n} x_{i} x_{j} x\right) x^{n-3}+ \\
& i \neq 2 j \neq 2 \quad k \neq 2 \text {, }
\end{aligned}
$$

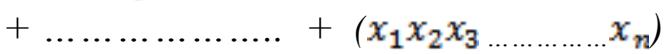

$$
\begin{aligned}
& \prod_{i=0}^{n}\left(x-x_{i}\right)=\left(x-x_{0}\right)\left(x-x_{1}\right)\left(x-x_{2}\right) \ldots \ldots \ldots\left(x-x_{n-1}\right) \\
& =x^{n}+\left(\sum_{i=0}^{n-1} x_{i}\right) x^{n-1}+\left(\sum_{i=0}^{n-2} \sum_{j=0}^{n} x_{i} x_{j}\right) x^{n-2}+ \\
& i \neq n \quad i \neq n j \neq n \\
& \begin{array}{cccc}
\sum_{i=0}^{n-3} & \sum_{j=0}^{n} \sum_{k=0}^{n} & \left.x_{i} x_{j} x_{k}\right) x^{n-3}+\ldots \ldots \ldots \ldots+\left(x_{1} x_{2} x_{3} \ldots \ldots \ldots \ldots . . . x_{n}\right) \\
i \neq n & j \neq n & k \neq n
\end{array}
\end{aligned}
$$

Therefore, equation (2.14) becomes

$$
\begin{aligned}
& y=C_{0}\left[x^{n}+\left(\sum_{i=0}^{n} x_{i}\right) x^{n-1}+\left(\sum_{i=0}^{n} \quad \sum_{j=0}^{n} x_{i} x_{j}\right) x^{n-2}+\right. \\
& i \neq 0 \quad i \neq 0 \quad j \neq 0 \\
& \left(\sum_{i=0}^{n} \sum_{j=0}^{n} \sum_{k=0}^{n} x_{i} x_{j} x_{k}\right) x^{n-3}+ \\
& i \neq 0 \quad j \neq 0 \quad k \neq 0 \\
& +\left(\sum_{i_{1}=0}^{n} \quad \sum_{i_{2}=0}^{n} \ldots \ldots \ldots \sum_{i_{r-1}=0}^{n} x_{i_{1}} x_{i_{2}} \ldots \ldots x_{i_{r-1}}\right) x^{n-r}+ \\
& i_{1} \neq 0, i_{2} \neq 0, \ldots \ldots, i_{r-1} \neq 0 \\
& \left..+\left(x_{1} x_{2} x_{3} \ldots \ldots \ldots \ldots x_{n}\right)\right]+ \\
& C_{1}\left[x^{n}+\left(\sum_{i=0}^{n} x_{i}\right) x^{n-1}+\left(\sum_{i=0}^{n} \quad \sum_{j=0}^{n} x_{i} x_{j}\right) x^{n-2}+\right. \\
& i \neq 1 \quad i \neq 1 \quad j \neq 1 \\
& \left(\sum_{i=0}^{n} \quad \sum_{j=0}^{n} \sum_{k=0}^{n} x_{i} x_{j} x_{k}\right) x^{n-3}+ \\
& i \neq 1 \quad j \neq 1 \quad k \neq 1 \\
& \left.+\left(\sum_{i_{1}=0}^{n} \sum_{i_{2}=0}^{n} \ldots \ldots \ldots \sum_{i_{r-1}=0}^{n} x_{i_{1}} x_{i_{2}} \ldots \ldots x_{i_{r-1}}\right) x^{n-r}+\left(x_{1} x_{2} x_{3} \ldots \ldots \ldots \ldots x_{n}\right)\right] \\
& i \neq 0, i_{2} \neq 0, \ldots \ldots \ldots, i_{r-1} \neq 0 \\
& +C_{2}\left[x^{n}+\left(\sum_{i=0}^{n} x_{i}\right) x^{n-1}+\left(\sum_{i=0}^{n} \quad \sum_{j=0}^{n} x_{i} x_{j}\right) x^{n-2}+\right. \\
& i \neq 2 \quad i \neq 2 \quad j \neq 2 \\
& \begin{array}{cll}
\left(\sum_{i=0}^{n} \sum_{j=0}^{n} \sum_{k=0}^{n}\right. & \left.\left.x_{i} x_{j} x_{k}\right) x^{n-3}+\ldots \ldots \ldots \ldots+\left(x_{1} x_{2} x_{3} \ldots \ldots \ldots \ldots x_{n}\right)\right] \\
i \neq 2 \quad j \neq 2 & k \neq 2
\end{array}
\end{aligned}
$$




$$
\begin{aligned}
& C_{n}\left[\mathrm{x}^{\mathrm{n}}+\left(\sum_{i=0}^{n} x_{i}\right) x^{n-1}+\left(\sum_{i=0}^{n} \sum_{j=0}^{n} x_{i} x_{j}\right) x^{n-2}+\right. \\
& i \neq n \quad i \neq n \quad j \neq n
\end{aligned}
$$

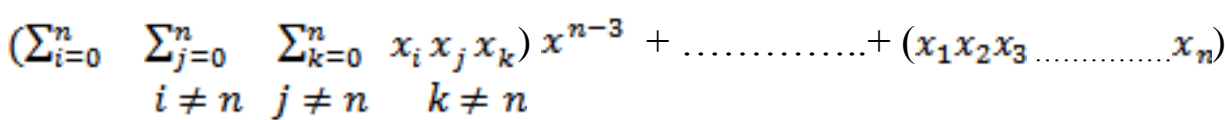

This can be arranged as

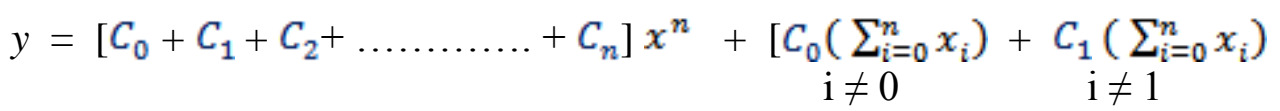

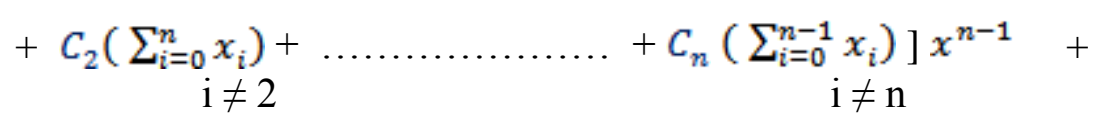

$$
\begin{aligned}
& {\left[C_{0}\left(\sum_{i=0}^{n} \sum_{j=0}^{n} x_{i} x_{j}\right)+C_{1}\left(\sum_{i=0}^{n} \sum_{j=0}^{n} x_{i} x_{j}\right)+C_{2}\left(\sum_{i=0}^{n} \sum_{j=0}^{n} x_{i} x_{j}\right)\right.} \\
& i \neq 0 \quad j \neq 0 \quad i \neq 1 \quad j \neq 1 \text {. } \\
& \mathrm{i} \neq \mathrm{j} \quad \mathrm{i} \neq \mathrm{j} \quad \mathrm{i} \neq \mathrm{j} \\
& \left.+\ldots \ldots \ldots \ldots \ldots \ldots+\mathrm{C}_{n}\left(\sum_{i=1}^{n} \sum_{j=1}^{n} x_{i} x_{j}\right)\right] x^{n-2}+ \\
& i \neq n \quad j \neq n \\
& \mathrm{i} \neq \mathrm{j} \\
& +\left[\left(x_{1} x_{2} x_{3} \ldots \ldots \ldots x_{n}\right)+\left(x_{0} x_{2} x_{3} \ldots \ldots \ldots x_{n}\right)+\ldots \ldots+\left(x_{0} x_{2} \ldots \ldots \ldots x_{n-1}\right)\right]
\end{aligned}
$$

Now writing

$$
\mathrm{S}_{\mathrm{r}}(1)=\sum_{i=0}^{n} x_{i} \quad, \quad \mathrm{~S}_{\mathrm{r}}(2)=\sum_{i=0}^{n} \sum_{j=0}^{n} x_{i} x_{j}
$$

$\mathrm{i} \neq \mathrm{r}$

$$
\mathrm{i} \neq \mathrm{r}, \mathrm{j} \neq \mathrm{r}, \mathrm{i} \neq \mathrm{j}
$$

$$
\begin{gathered}
S_{r_{1} r_{2} \ldots \ldots m \ldots m} r_{p}=\sum_{i_{1}=0}^{n} \sum_{i_{2}=0}^{n} \ldots \ldots \ldots \sum_{i_{p}=0}^{n} x_{i_{1}} x_{i_{2}} \ldots \ldots x_{i_{p}}, \\
i_{1} \neq r_{1}, i_{2} \neq r_{1} \ldots \ldots \ldots i_{p} \neq r_{p} \\
i_{1} \neq i_{2} \neq \ldots \ldots \ldots \neq i_{p}
\end{gathered}
$$

one can finally obtain that

$$
\begin{aligned}
& y=\left(\sum_{\mathrm{i}=0}^{\mathrm{n}} \mathrm{C}_{\mathrm{i}}\right) x^{n}+\left\{\sum_{\mathrm{i}=0}^{\mathrm{n}} \mathrm{C}_{\mathrm{i}} \mathrm{S}_{\mathrm{i}}(1)\right\} x^{n-1}+\left\{\sum_{\mathrm{i}=0}^{\mathrm{n}} \mathrm{C}_{\mathrm{i}} \mathrm{S}_{\mathrm{i}}(2)\right\} x^{n-2}+\left\{\sum_{\mathrm{i}=0}^{\mathrm{n}} \mathrm{C}_{\mathrm{i}} \mathrm{S}_{\mathrm{i}}(3)\right\} x^{n-3} \\
& +\left\{\sum_{\mathrm{i}=0}^{\mathrm{n}} \mathrm{C}_{\mathrm{i}} S_{i}(r)\right\} x^{n \text { 棡 } r}+ \\
& +\left\{\sum_{i=0}^{n} C_{i} S_{i}(n \text { 焵 } 1)\right\} x+\sum_{i=0}^{n} C_{i} S_{i}(n)
\end{aligned}
$$


Therefore, $y$ is of the form

$$
\begin{aligned}
y= & D_{0} x^{n}+D_{1} x^{n-1}+D_{2} x^{n-2}+D_{3} x^{n-3}+\ldots \ldots \ldots \ldots \ldots \ldots \ldots \ldots \ldots \ldots \ldots \ldots \ldots \ldots \ldots \ldots \ldots \ldots \ldots \ldots \\
& +\ldots \ldots \ldots \ldots \ldots \ldots \ldots+D_{n-1} x+D_{n}
\end{aligned}
$$

where

$D_{0}=\sum_{i=0}^{n} C_{\tilde{i}}$,

$$
\begin{aligned}
& D_{1}=\sum_{i=0}^{n} C_{i} S_{i}(1) \text {, } \\
& D_{2}=\sum_{i=0}^{n} C_{i} S_{i}(2) \text {, } \\
& D_{3}=\sum_{i=0}^{n} C_{i} S_{i}(3) \text {, } \\
& D_{r}=\sum_{i=0}^{n} C_{i} S_{i}(r), \\
& D_{n-1}=\sum_{i=0}^{n} C_{i} S_{i}(n-1) \text {, } \\
& D_{n}=\sum_{i=0}^{n} C_{i} S_{i}(n) \text {. }
\end{aligned}
$$

This is the required formula for representation of numerical data by a polynomial curve.

\section{Numerical Example of Application}

The following table shows the data on total population of India corresponding to the years:

\begin{tabular}{|c|c|c|c|c|c|}
\hline Year & 1971 & 1981 & 1991 & 2001 & 2011 \\
\hline Total Population & 548159652 & 683329097 & 846302688 & 1027015247 & 1210193422 \\
\hline
\end{tabular}

Taking 1971 as origin and changing scale by 1/10, one can obtain the following table for independent variable $x$ (representing time) and $y=f(x)$ (representing total population of India):

\begin{tabular}{|c|c|c|c|c|c|}
\hline Year & 1971 & 1981 & 1991 & 2001 & 2011 \\
\hline$x_{i}$ & 0 & 1 & 2 & 3 & 4 \\
\hline$y_{i}=f(x)$ & 548159652 & 683329097 & 846302688 & 1027015247 & 1210193422 \\
\hline
\end{tabular}

Now here $x_{0}=0, x_{1}=1, x_{2}=2, x_{3}=3, x_{4}=4$

$$
\begin{aligned}
& y_{0}=f\left(x_{0}\right)=548159652, y_{1}=f\left(x_{1}\right)=683329097, f\left(x_{2}\right)=846302688, f\left(x_{3}\right)=1027015247, \\
& f\left(x_{4}\right)=1210193422 .
\end{aligned}
$$

Thus,

$$
\begin{aligned}
C_{0}= & \frac{548159652}{(0-1)(0-2)(0-3)(0-4)}=22839985.5, \\
C_{1}= & \frac{683329097}{(1-0)(1-2)(1-3)(1-4)}=-113888182.83, \\
& C_{2}=\frac{846302688}{(2-0)(2-1)(2-3)(2-4)}=211575672, \\
C_{3}= & \frac{1027015247}{(3-0)(3-1)(3-2)(3-4)}=-171169207.83,
\end{aligned}
$$




$$
C_{4}=\frac{1210193422}{(4-0)(4-1)(4-2)(4-3)}=50424725.91
$$

Now,

$$
\begin{aligned}
& D_{0}=\sum_{i=0}^{n} C_{i}=C_{0}+C_{1}+C_{2}+C_{3}+C_{4}=-217007.25 \text {, } \\
& D_{1}=\sum_{i=0}^{n} C_{i} S_{i}(1)=C_{0}\left(x_{1}+x_{2}+x_{3}+x_{4}\right)+C_{1}\left(x_{0}+x_{2}+x_{3}+x_{4}\right)+C_{2}\left(x_{0}+x_{1}+x_{3}+x_{4}\right) \\
& +C_{3}\left(x_{0}+x_{1}+x_{2}+x_{4}\right)+C_{4}\left(x_{0}+x_{1}+x_{2}+x_{3}\right) \text {, } \\
& =22839985.5 \times 10+(-113888182.83) \times 9+211575672 \times 8+(-171169207.83) \times 7 \\
& +50424725.91 \times 6 \\
& =375486.2 \text {, } \\
& D_{2}=\sum_{i=0}^{n} C_{i} S_{i}(2) \\
& =C_{0}\left(x_{1} x_{2}+x_{1} x_{3}+x_{1} x_{4}+x_{2} x_{3}+x_{2} x_{4}+x_{3} x_{4}\right)+C_{1}\left(x_{0} x_{2}+x_{0} x_{3}+x_{0} x_{4}+x_{2} x_{3}+x_{2} x_{4}\right. \\
& \left.+x_{3} x_{4}\right)+C_{2}\left(x_{0} x_{1}+x_{0} x_{3}+x_{0} x_{4}+x_{1} x_{3}+x_{1} x_{4}+x_{3} x_{4}\right)+C_{3}\left(x_{0} x_{1}+x_{0} x_{2}+x_{0} x_{4}\right. \\
& \left.+x_{1} x_{2}+x_{1} x_{4}+x_{2} x_{4}\right)+C_{4}\left(x_{0} x_{1}+x_{0} x_{2}+x_{0} x_{3}+x_{1} x_{2}+x_{1} x_{3}+x_{2} x_{3}\right) \\
& =22839985.5 \times 35+(-113888182.83) \times 26+211575672 \times 19+ \\
& (-171169207.83) \times 14+50424725.91 \times 11 \\
& =16547582.3 \text {, } \\
& D_{3}=\sum_{i=0}^{n} C_{i} S_{i}(3) \\
& =C_{0}\left(x_{1} x_{2} x_{3}+x_{1} x_{3} x_{4}+x_{2} x_{3} x_{4}+x_{1} x_{2} x_{4}\right)+C_{1}\left(x_{0} x_{2} x_{3}+x_{0} x_{2} x_{4}+x_{0} x_{3} x_{4}\right. \\
& \left.+x_{2} x_{3} x_{4}\right)+C_{2}\left(x_{0} x_{1} x_{3}+x_{0} x_{3} x_{4}+x_{0} x_{1} x_{4}+x_{1} x_{3} x_{4}\right)+C_{3}\left(x_{0} x_{1} x_{2}+x_{0} x_{2} x_{4}\right. \\
& \left.+x_{0} x_{1} x_{4}+x_{1} x_{2} x_{4}\right)+C_{4}\left(x_{0} x_{1} x_{2}+x_{0} x_{1} x_{3}+x_{0} x_{2} x_{3}+x_{1} x_{2} x_{3}\right) \\
& =22839985.5 \times 50+(-113888182.83) \times 24+211575672 \times 12+ \\
& (-171169207.83) \times 8+50424725.91 \times 6 \\
& =-119214356 \text {, } \\
& D_{4}=\sum_{i=0}^{n} C_{i} S_{i}(4) \\
& =C_{0}\left(x_{1} x_{2} x_{3} x_{4}\right)+C_{1}\left(x_{0} x_{2} x_{3} x_{4}\right)+C_{2}\left(x_{0} x_{1} x_{3} x_{4}\right)+C_{3}\left(x_{0} x_{1} x_{2} x_{4}\right)+C_{4}\left(x_{0} x_{1} x_{2} x_{3}\right) \\
& =22839985.5 \times 24+(-113888182.83) \times 0+211575672 \times 0+(-171169207.83) \times \\
& 0+50424725.91 \times 0 \\
& =548159652
\end{aligned}
$$$$
\therefore y=f(x)=D_{0} x^{4}+D_{1} x^{3}+D_{2} x^{2}+D_{3} x+D_{4}
$$$$
=-217007.25 x^{4}-375486.2 x^{3}+16547582.3 x^{2}+119214356 x+548159652
$$

Hence, the equation of the polynomial curve that represent the given numerical data is $y=f(x)=-217007.25 x^{4}-375486.2 x^{3}+16547582.3 x^{2}+119214356 x+548159652$

The values of the function $y=f(x)$ at the obse4rved values of $x$ yielded by this polynomial curve are as follows::

$$
\begin{aligned}
f(0) & =-217007.25 \times 0-375486.2 \times 0+16547582.3 \times 0+119214356 \times 0+548159652 \\
& =548159652 \\
f(1) & =-217007.25 \times 1-375486.2 \times 1+16547582.3 \times 1+119214356 \times 1+548159652 \\
& =683329097 \\
f(2) & =-217007.25 \times 2^{4}-375486.2 \times 2^{3}+16547582.3 \times 2^{2}+119214356 \times 2+548159652
\end{aligned}
$$




$$
\begin{aligned}
& =846302687.6 \\
& f(3)=-217007.25 \times 3^{4}-375486.2 \times 3^{3}+16547582.3 \times 3^{2}+119214356 \times 3+548159652 \\
& =1027015246.1 \\
& f(4)=-217007.25 \times 4^{4}-375486.2 \times 4^{3}+16547582.3 \times 4^{2}+119214356 \times 4+548159652 \\
& =1210193432 \text {. }
\end{aligned}
$$

\section{Conclusion}

The formula described by equation (2.17) can be used to represent a given set of numerical data on a pair of variables, by a polynomial. The degree of the polynomial is one less than the number of pairs of observations.

The polynomial that represents the given set of numerical data can be used for interpolation at any position of the independent variable lying within its two extreme values.

It has been found from the study that for given $(n+1)$ pairs of values

$$
\left(x_{0}, y_{0}\right),\left(x_{1}, y_{1}\right),\left(x_{2}, y_{2}\right),\left(x_{3}, y_{3}\right), \ldots \ldots \ldots \ldots \ldots,\left(x_{n}, y_{n}\right)
$$

on a pair of variables $X \& Y$, it is possible to express $Y$ as a polynomial in $X$ of degree $n$ that can represent the given $(n+1)$ pairs of values. Similarly, it is possible to express $X$ as a polynomial in $Y$ of degree $n$ that can represent the given $(n+1)$ pairs of values. Thus, the formula $(2.17)$ can be applicable, by interchanging the variables in it, in inverse interpolation also.

\section{Reference:}

Bathe K. J. \& Wilson E. L., Numerical Methods in Finite Element Analysis, Prentice-Hall, Englewood Cliffs, NJ, 1976.

Erdos P. \& Turan P., On Interpolation II: On the Distribution of the Fundamental Points of Lagrange and Hermite Interpolation. The Annals of Mathematics, 2nd Ser, Vol. 39, No. 4, pp. 703-724, 1938

Hummel P. M., A Note on Interpolation (in Mathematical Notes). American Mathematical Monthly, Vol. 54, No. 4, pp. 218-219, 1947.

Jan K. Wisniewski, Note on Interpolation (in Notes). Journal of the American Statistical Association Vol. 25, No. 170, pp. 203-205, 1930.

Mills T. M., An introduction to analysis by Lagrange interpolation. Austral. Math. Soc. Gaz., Vol. 4, No. 1, pp. $10-18,1977$.

Neale E. P. \& Sommerville D. M. Y., A Shortened Interpolation Formula for Certain Types of Data. Journal of the American Statistical Association, Vol. 19, No. 148, pp. 515-517, 192 
Quadling D. A., Lagrange's Interpolation Formula. The Mathematical Gazette, Vol. L, No. 374, pp. 372-375, 1966

Revers \& Michael Bull, On Lagrange interpolation with equally spaced nodes. Austral. Math. Soc MathSciNet., Vol. 62, No. 3, pp. 357-368, 2000.

Traub J. F., On Lagrange-Hermite Interpolation. Journal of the Society for Industrial and Applied Mathematics, Vol. 12, No. 4, pp. 886-891, 1964.

Whittaker E. T. \& Robinson G., Lagrange's Formula of Interpolation, The Calculus of Observations: A Treatise on Numerical Mathematics, 4th ed.. §17, New York: Dover, pp. 28 - 30, 1967. 